\title{
Líquen plano hipertrófico. A propósito de un caso
}

\author{
Hypertrophic lichen planus. A case report
}

\section{Leticia Santos Rey¹, Yésica Expósito Casabella ${ }^{1}$, Benigno Monteagudo Sánchez², Carlos M. Neira de Paz , Abián Mosquera Fernández $^{4}$}

\author{
${ }^{1}$ Grado en Podología. Universidade da Coruña \\ leticia.santos.rey@hotmail.com; je.casabella@gmail.com \\ ${ }^{2}$ Licenciado en Medicina y Cirugía. Servicio de Dermatología. Complejo Hospitalario Universitario de Ferrol. Área \\ Sanitaria de Ferrol. Servicio Gallego de Salud. \\ benigno.monteagudo.sanchez@sergas.es \\ ${ }^{3}$ Licenciado en Medicina y Cirugía. Servicio de Anatomía Patológica. Complejo Hospitalario Universitario de \\ Ferrol. Área Sanitaria de Ferrol. Servicio Gallego de Salud. \\ carlos.manuel.neira.de.paz@sergas.es \\ ${ }^{4}$ Profesor colaborador doctor. Departamento de Ciencias de la Salud. Facultad de Enfermería y Podología. Universi- \\ dade da Coruña, Campus de Ferrol. \\ abian@udc.es
}

Correspondencia:

Dr. Benigno Monteagudo Sánchez

Servicio de Dermatologia. Hospital Naval de Ferrol

Avda. de la Residencia $\mathrm{s} / \mathrm{n}$

E-15405 Ferrol (A Coruña)

Correo electrónico: benigno.monteagudo.sanchez@sergas.es

Fecha de recepción: 1 de julio de 2014

Fecha de aceptación: 2 de octubre de 2014

Los autores declaran no tener ningún tipo de interés económico o comercial.

\section{RESUMEN}

El liquen plano es una dermatosis inflamatoria crónica relativamente frecuente en adultos de edad media y que normalmente afecta a la piel, membranas mucosas, pelo y uñas bajo diferentes patrones morfológicos motivo por el que su diagnóstico puede presentar cierta dificultad. Para un diagnóstico correcto es imprescindible el examen físico y complementario.

La etiología del liquen plano es desconocida aunque se han postulado diferentes mecanismos etiopatogénicos de los que el inmune es el más prevalente. El liquen plano hipertrófico constituye una de sus variedades morfológicas que se caracteriza por localizarse a nivel de las extremidades y por ser muy pruriginoso. En aquellos pacientes con una presentación clínica atípica es además necesario realizar un seguimiento por la posibilidad de malignización que se ha descrito en la literatura científica. Describimos el caso clínico de una mujer de 70 años con liquen plano hipertrófico a nivel de la extremidad inferior que acude al Servicio de Dermatología del Hospital Naval de Ferrol.

Palabras clave: liquen plano; liquen plano hipertrófico; tumor.

\begin{abstract}
Lichen planus is a chronic and relatively common inflammatory dermatosis in middle aged adults that usually affects the skin, mucous membranes, hair and nails under different morphological patterns reason for why its diagnosis can present some difficulty. To perform an accurate diagnosis a physical and complementary exam is essential. The etiology of lichen planus is unknown although different etiologic mechanisms have been postulated of which the immune is the most prevalent. Hypertrophic lichen planus constitutes one of its morphological varieties characterized by a very itchy rash and by being localized at limbs level. In patients with an atypical clinical presentation a followup is also necessary to monitor the possibility of malignant transformation that has been described in the scientific literature. We report the case of a 70 year old woman with hypertrophic lichen planus affecting the lower extremities admitted to the outpatient clinic in the Dermatology Department of the Naval Hospital of Ferrol.
\end{abstract}

Keywords: lichen planus; hypertrophic lichen planus; tumor.

Sumario: 1. Introducción. 2. Caso clínico. 3. Discusión. Bibliografía.

Referencia normalizada: Santos Rey, L., Expósito Casabella, Y., Monteagudo Sánchez, B., Neira de Paz, C., Mosquera Fernández, A. Líquen plano hipertrófico. A propósito de un caso. Rev. Int. Cienc. Podol. 2015; 9(2): 85-88. 


\section{INTRODUCCIÓN}

El liquen plano (LP) es una dermatosis crónica e inflamatoria de origen desconocido y patogenia autoinmunitaria que se ha relacionado con numerosas enfermedades dermatológicas y sistémicas (alopecia areata, psoriasis dermatitis atópica, hipertensión, diabetes mellitus, enfermedad tiroidea y cardiopatías) y que puede afectar tanto a la piel como a las mucosas en un $30 \%-70 \%$ de los $\operatorname{casos}^{1}$. A pesar de ello existen factores desencadenantes como algunos agentes infecciosos, especialmente la infección por el virus de la hepatitis $\mathrm{C}^{2-4}$. A nivel cutáneo suele presentarse como pápulas o placas violáceas de superficie plana y con un reticulado blanquecino característico. La afectación leve y temporal de la lámina ungueal se da entre el $1 \%$ y el $10 \%$ de los casos aunque en aproximadamente un $4 \%$ ocasiona un daño irreversible ${ }^{5}$. En España se ha detectado una asociación significativa entre el LP y la dislipemia ${ }^{6}$, de hecho se cree que la inflamación crónica presente en personas afectadas de liquen plano sería la responsable de su relación con la dislipemia según un estudio de casos y controles que tuvo por objeto evaluar los factores de riesgo cardiovascular incluidos en el documento Adult Treatment $\mathrm{Pa}$ nel III (ATP-III) ${ }^{7}$. La prevalencia exacta del LP es desconocida sin embargo, a nivel mundial, se estima que fluctua entre el $0,22 \%$ y el $5 \%{ }^{8}$. Su diagnóstico es clínico en las formas clásicas pero la biopsia es necesaria para confirmarlo y de utilidad ante presentaciones atípicas ${ }^{9}$. El liquen plano hipertrófico (LPH) es una variante caracterizada por la presencia de placas hiperqueratósicas de aspecto verrugoso, gruesas y muy pruriginosas que aparece generalmente en la cara anterior de las piernas y articulaciones interfalángicas siguiendo una distribución simétrica $^{8,10}$. Se trata de una forma clínica poco frecuente que afecta con mayor frecuencia a la raza negra y a pacientes inmunosuprimidos ${ }^{11}$.

\section{CASO CLÍNICO}

Mujer de 70 años de edad con antecedentes personales de cáncer de mama, hipertensión e hipercolesterolemia que consulta por unas lesiones cutáneas pruriginosas en ambas piernas de un año de evolución. En la exploración se aprecia una placa violácea con un reticulado blanquecino en superficie, localizada en la cara posterior de la pierna izquierda (Figura 1). Presentaba además otras lesiones de similares características pero menor tamaño dispuestas de forma bilateral en ambas extremidades superiores e inferiores y área lumbar.

La actitud terapéutica seguida fue la realización de una biopsia cuyo estudio histopatológico evidenció una hiperplasia epidérmica irregular. A nivel dérmico destacaba un infiltrado linfoide más pronunciado en el extremo de las crestas interpapilares que llegaba a afectar a la capa basal y la presencia de queratinocitos apoptóticos (Figura 2). Estos hallazgos fueron compatibles con la sospecha diagnóstica inicial de LPH. Se realizó tratamiento con hidroxicina oral $25 \mathrm{mg}$ al día y con propionato de clobetasol en crema al 0,1\% (una aplicación diaria en oclusión nocturna sobre las lesiones de mayor tamaño durante 20 días consecutivos y posteriormente otro mes más a días alternos). Con ello se logró la resolución de las lesiones activas y del prurito persistiendo únicamente una hiperpigmentación postinflamatoria residual.

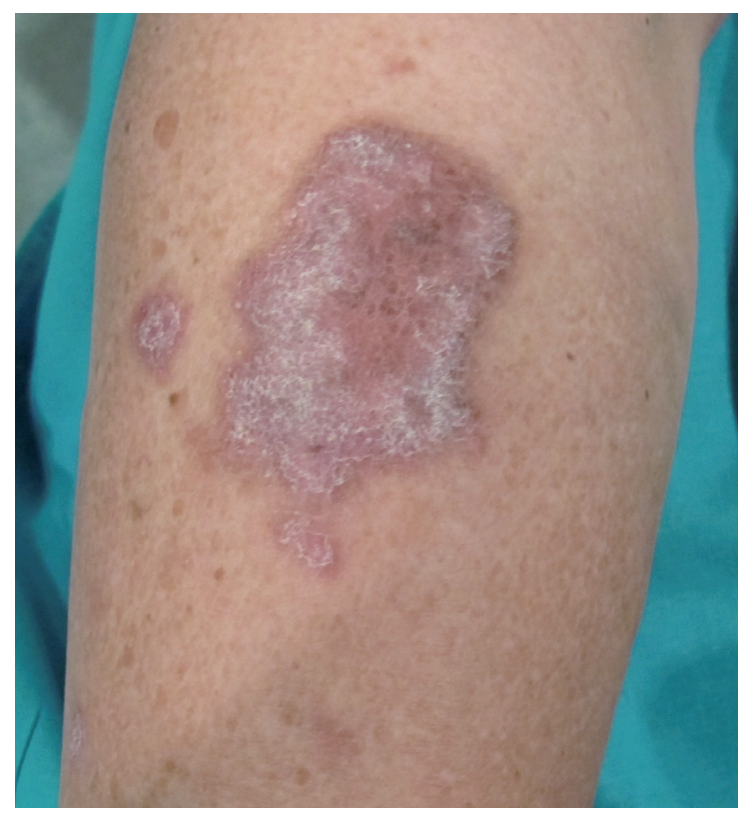

Figura. 1. Pápulas y placas violáceas, con un reticulado blanquecino en superficie en la cara posterior de la pierna izquierda. 


\section{DISCUSIÓN}

La prevalencia del LPH es desconocida. Existe un predominio en adultos y en el sexo femenino (el $65 \%$ de los casos se da en mujeres ${ }^{12}$ ), si bien en niños es poco común ${ }^{13}$. Las lesiones más características son las pápulas poligonales eritemato-violáceas con una red de finas líneas blanquecinas en su superficie llamadas estrías de Wickham. Suelen localizarse en las flexuras, las muñecas, la región lumbar y los tobillos. No es rara la afectación de las mucosas oral y genital, el cuero cabelludo y las uñas ${ }^{10,14}$. El diagnóstico diferencial principal son las erupciones liquenoides causadas por fármacos y las enfermedades papuloescamosas como la dermatitis atópica, la sífilis secundaria y las tubercúlides. En ocasiones puede confundirse con la psoriasis en gotas, la psoriasis ungueal o la psoriasis invertida o genita $1^{15}$.
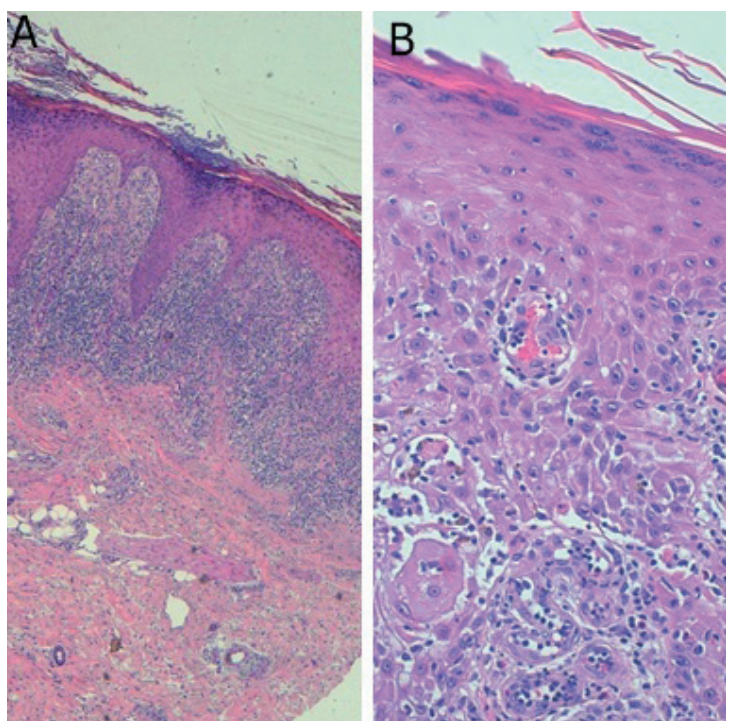

Figura 2. A-Hiperplasia epidérmica irregular e infiltrado linfoide dérmico (hematoxilina-eosina, $\times 40$ ). B-A mayor detalle, infiltrado linfoide más pronunciado en el extremo de las crestas interpapilares y presencia de queratinocitos apoptóticos (hematoxilina-eosina, $\times 200$ ).

El tratamiento depende de la gravedad y de la localización de las lesiones. Por norma general se utilizan corticoides en forma tópica, intralesional y vía oral. En determinadas oca- siones es preciso recurrir a tacrolimus tópico, ciclosporina, retinoides orales o a la fototerapia. También es frecuente la asociación de antihistamínicos orales para reducir el prurito ${ }^{10,16}$.

El LPH también llamado liquen plano verrugoso es una variante clínica del LP de predominio en varones. Parece que la éstasis venosa crónica puede contribuir a la aparición de esta dermatosis. Se manifiesta como placas gruesas hiperqueratósicas y descamación blanquecina sobre todo en las zonas pretibiales, los tobillos, y el dorso de los pies y las manos. Es intensamente pruriginoso y tiende a la cronicidad por el rascado repetitivo con una media de duración de 6 años. De igual forma como ha quedado demostrado en el caso expuesto no son infrecuentes la hiperpigmentación o las cicatrices residuales. En lesiones de largo tiempo de evolución se han descrito casos de desarrollo de cuernos cutáneos, queratoacantoma o incluso de malignización (carcinoma epidermoide y carcinoma de células escamosas) ${ }^{17,18}$; de hecho estudios epidemiológicos llevados a cabo durante los últimos 20 años estiman que la tasa de potencial malignización del liquen plano ronda el $0,27 \%$ de ahí la importancia de seguir de cerca la evolución de aquellos pacientes con $\mathrm{LPH}^{19}$. En nuestro caso el diagnóstico se basó, además de en una histopatología compatible, en una clínica característica basada en una placa engrosada con un reticulado blanquecino en superficie, lesiones de menor tamaño en su proximidad típicas de LP y la localización en pierna. El diagnóstico diferencial del LPH debe de realizarse con el liquen simple crónico (eczema crónico), el prurigo nodular secundario al rascado crónico, la amiloidosis cutánea (liquen amiloideo) y el sarcoma de Kaposi ${ }^{15}$.

Dado el grosor de las lesiones del LPH, es preciso recurrir a tratamiento con corticoides tópicos potentes en forma oclusiva o el acetónido de triamcinolona intralesional en concentraciones de 10 a $20 \mathrm{mg} / \mathrm{ml}$, normalmente acompañados de un antihistamínico oral para aliviar el prurito ${ }^{10}$. En este caso se utilizaron los corticoides tópicos con cura oclusiva nocturna. Dicha técnica favorece la penetración y aumenta la eficacia del corticosteroide, pero el tiempo de oclusión no debe exceder las 12 horas diarias para no incrementar en exceso los efectos adversos locales como la atrofia cutánea. 
En conclusión, describimos un nuevo caso de LPH a nivel de la extremidad inferior para destacar las características clínicas en las que se basa su diagnóstico, la eficacia de los corticoi- des tópicos en cura oclusiva en su tratamiento y el riesgo potencial de malignización de las lesiones cronificadas.

\section{BIBLIOGRAFÍA}

1. Giménez García R, Pérez Castrillón JL. Liquen plano y enfermedades asociadas: estudio clinicoepidemiológico. Actas Dermosifiliogr. 2004; 95(3):154-60.

2. Asaad T, Samdani AJ. Association of lichen planus with hepatitis C virus infection. Ann Saudi Med. 2005; 25(3): 243-6.

3. Ukonu AB, Augustine $U$. The prevalence of hepatitis $C$ virus among lichen planus patients and its clinical pattern at the University of Abuja Teaching Hospital, Gwagwalada, Abuja, Nigeria. Glob J Health Sci. 2012; 4(5): 113-9. doi: 10.5539/gjhs.v4n5p113.

4. Ponce M, Mendoza R, Paredes G. Liquen plano hipertrófico generalizado. Dermatol Peru. 2010; 20(4):240-4.

5. Allevato MA, Claros G, Donatti L, Juárez MA, Cabrera HN. Liquen córneo hipertrófico con compromiso ungular. Arch Argent Dermatol. 2008; 58:61-4.

6. Arias Santiago S, Buendía Eisman A, Aneiros Fernández J, Girón Prieto MS, Gutiérrez Salmerón MT, García Mellado V, et al. Lipid levels in patients with lichen planus: a case-control study. J Eur Acad Dermatol Venereol. 2011; 25:1398-1401. doi: 10.1111/j.1468-3083.2011.03983.x.

7. Arias Santiago S, Buendía Eisman A, Aneiros Fernández J, Girón Prieto MS, Gutiérrez Salmerón MT, García Mellado V, et al. Cardiovascular risk factors in patients with lichen planus. Am J Med. 2011; 124(6):543-8. doi: 10.1016/j.amjmed.2010.12.025.

8. Gorouhi F, Davari P, Fazel N. Cutaneous and mucosal lichen planus: a comprehensive review of clinical subtypes, risk factors, diagnosis and prognosis. ScientificWorldJournal. 2014, 2014:742826. doi:10.1155/2014/742826.

9. Usatine RP, Tinitigan M. Diagnosis and treatment of lichen planus. Am Fam Physician. 2011; 84(1):5360.

10. Sánchez-Saldaña L, Cabanillas Becerra JJ, Ponce Rodríguez MS, Salluca A. Liquen plano. Dermatol Peru. 2011; 21(1):27-44.

11. Bordel Gómez MT. Liquen plano hipertrófico infantil. An Pediatr (Barc). 2006; 64(4):392-8.

12. Martínez Casimiro L, Vilata Corell JJ. Liquen plano. Med Cutan Iber Lat Am. 2008; 36(5):223-31.

13. Handa S, Sahoo B. Childhood lichen planus: a study of 87 cases. Int J Dermatol. 2002; 41(7):423-7.

14. Le Cleach L, Chosidow O. Clinical Practice. Lichen planus. N Engl J Med. 2012; 366(8):723-32. doi: 10.1056/NEJMcp1103641.

15. Monteagudo Sánchez B, Rodríguez González L, León Muiños E, Ortiz Rey JA. Sífilis secundaria en paciente infectado por el VIH. Enferm Infecc Microbiol Clin. 2005; 23(3):175-6.

16. Wörheide J, Bonsmann G, Kolde G, Hamm H. Plattenepithelkarzinom auf dem boden eines lichen ruber hypertrophicus an der glans penis. Hautartz.1991; 42(2):112-5.

17. Sharma VK, Achar A, Raman M, Singh MK. Multiple cutaneous horns overlying lichen planus hypertrophicus. Br J Dermatol. 2001; 144(2):424-5.

18. Schirren CG, Schmoeckel C. Lichen ruber hypertrophicus with verrucous carcinoma. Hautarzt. 1992; 43(11):707-10.

19. Krasowska D, Kozlowicz K, Kowal M, Kurylcio A, Budzynska J, Polkowski W, et al. Twice malignant transformation of hypertrophic lichen planus. Ann Agric Environ Med. 2012; 19(4):787-9. 